\title{
An Online Gaming Testbed for Peer-to-Peer Architectures
}

\section{Max Lehn, Christof Leng, Robert Rehner, Tonio Triebel, Alejandro Buchmann}

\section{Introduction}

Goal: Provide a scalable testbed for P2P gaming mechanisms

Requirements:

- Gameplay representing a real game

- Attractive game to real players, which provide the reference behavior

- Simple enough for focusing on the important aspects

- Run in a real network as well as in a deterministic emulated network environment

- Resource-efficient for a good simulation scalability

- Well-defined and flexible interfaces to facilitate the replacement of network components

\section{Bots}

- Reproducible game workload generation

- Simulate human user behavior

- Implementations using finite state machines (FSM) or behavior trees (BT)

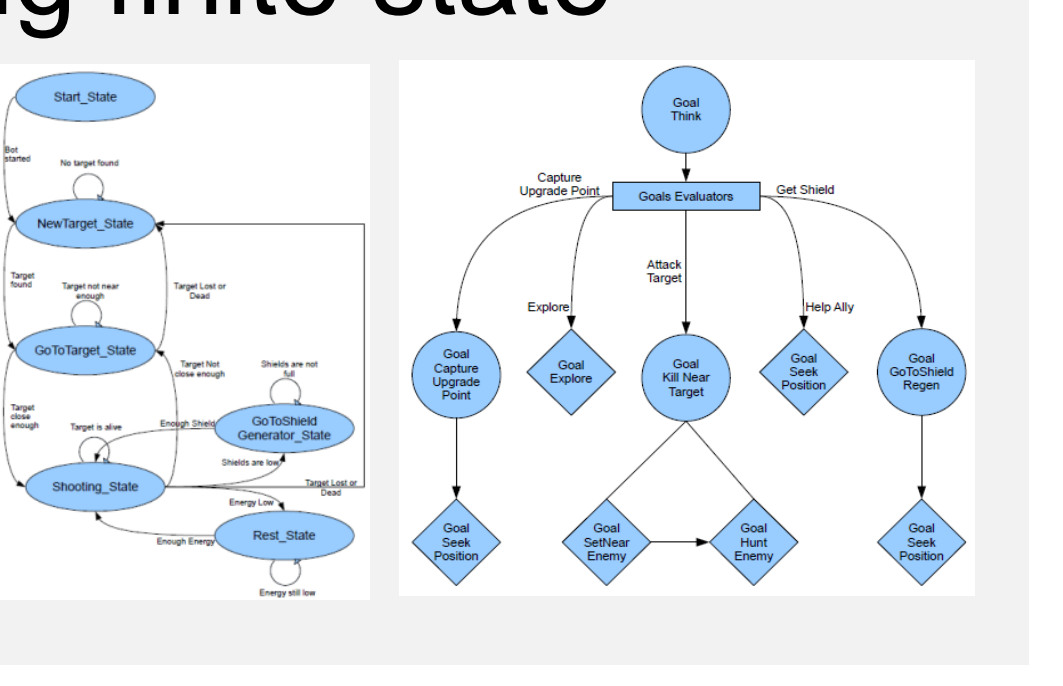

\section{Gameplay}

- Planet PI4 is a prototype of a 3D realtime massively multiplayer online game

- Competing teams

- Asteroid field sets the effective game world: parametrizable, random-generated

- Points of interest (POI): bases, upgrade points

- Rewards for capturing bases

\section{Network: Spatial Multicast}

- Dissemination of game events in a spatial context

- Interest management and multicast

- Instances: VON [1],

pSense [2],

pSense-3D,

client/server

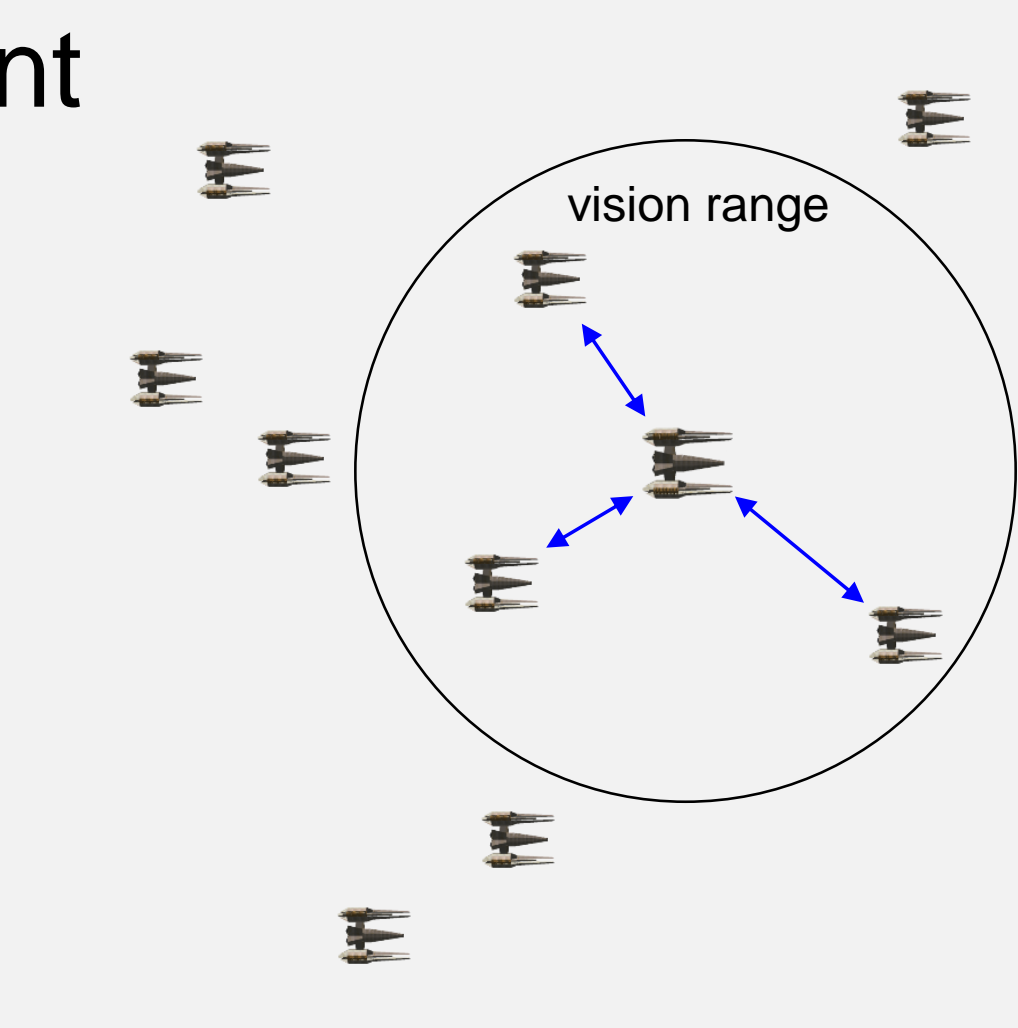

Simulation Engine [3]

- Discrete-event simulation

- Packet level network interface (UDP)

- Custom overlay simulator, alternatively ns-3

- Statistics interface, live plotting from SQLite database using gnuplot
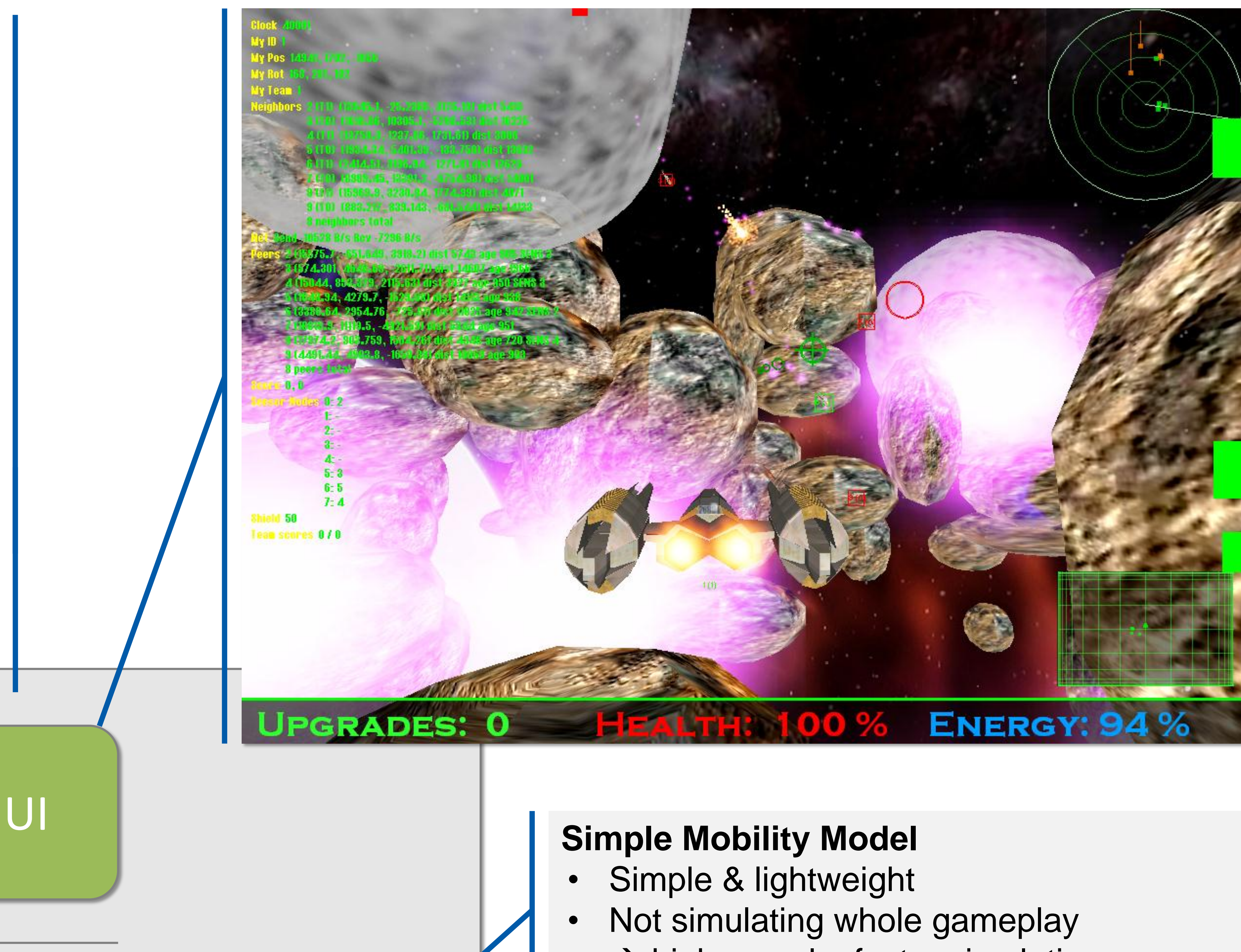

Bot

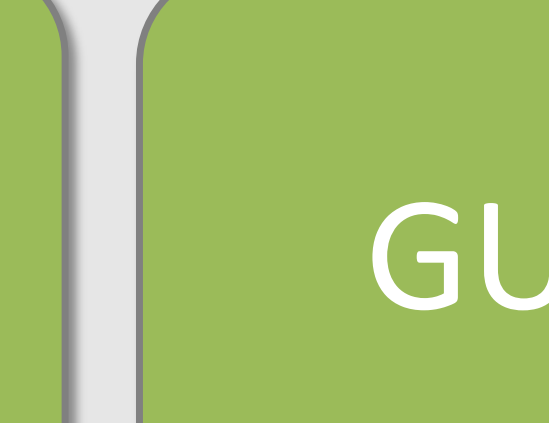

Planet PI4 Game

\section{Mobility} Model

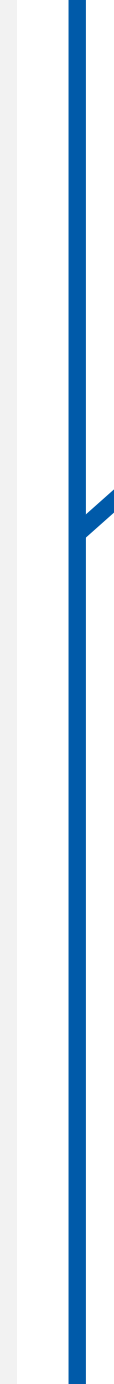

(1)
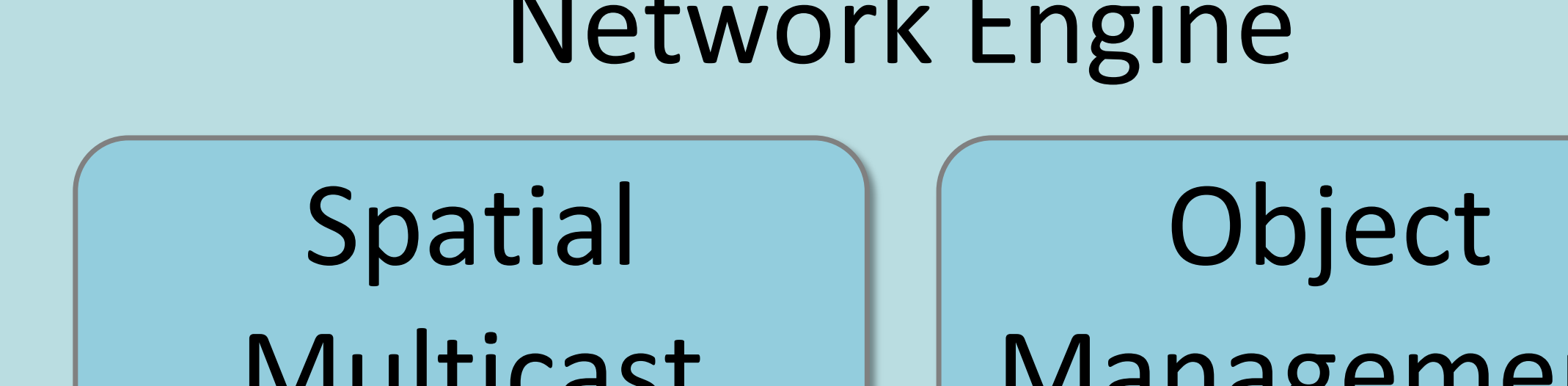

Multicast

Network Engine

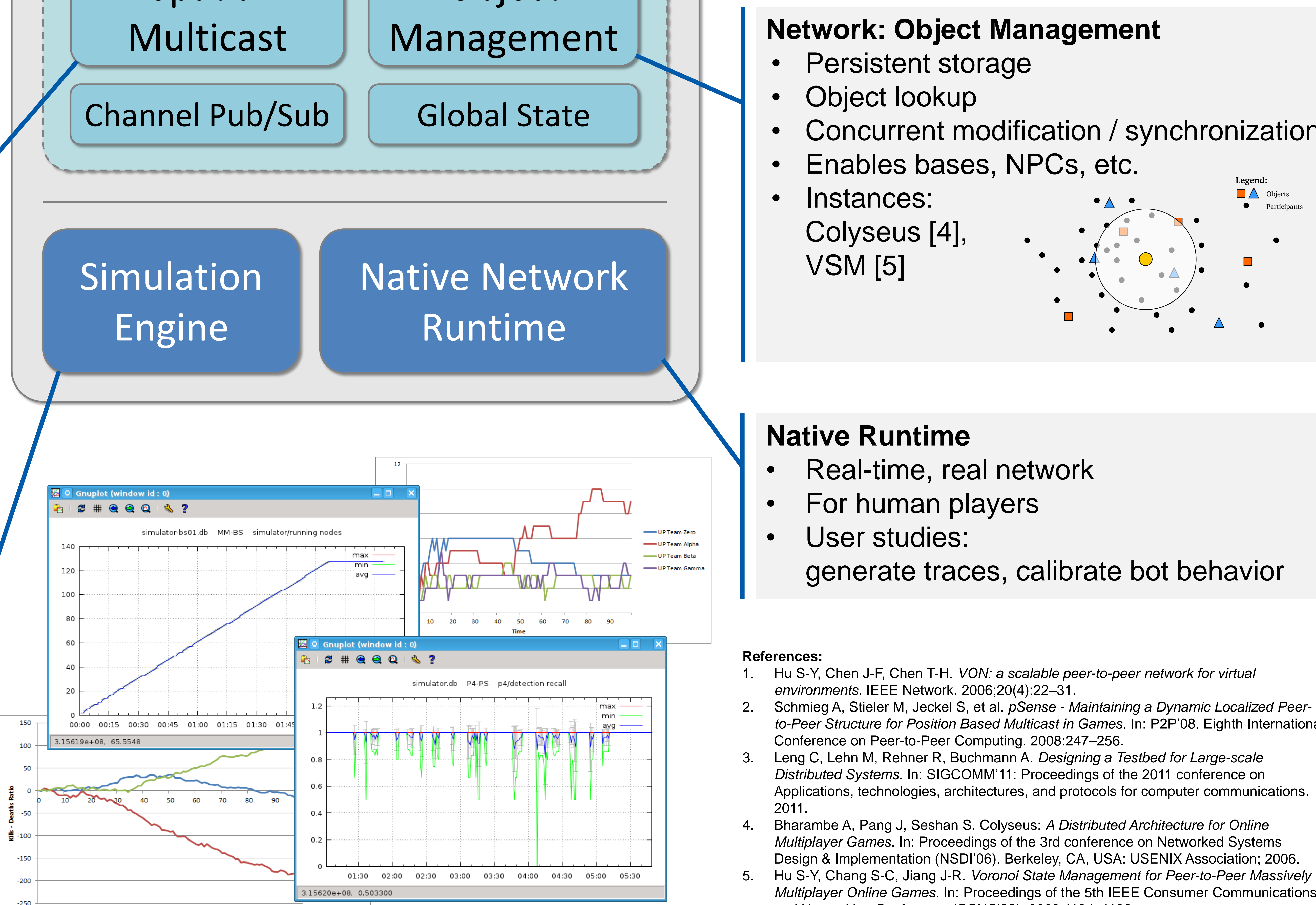

